\title{
Analysis of enhanced stimulated Brillouin scattering in silicon slot waveguides
}

\author{
Raphaël Van Laer, Bart Kuyken, Dries Van Thourhout and Roel Baets \\ Photonics Research Group, Ghent University-imec, Belgium \\ Center for Nano- and Biophotonics, Ghent University, Belgium *
}

compiled: October 23, 2013

\begin{abstract}
Stimulated Brillouin scattering has attracted renewed interest with the promise of highly tailorable integration into the silicon photonics platform. However, significant Brillouin amplification in silicon waveguides has yet to be shown. In an effort to engineer a structure with large photon-phonon coupling, we analyzed both forward and backward Brillouin scattering in high-index-contrast silicon slot waveguides. The calculations predict that gradient forces enhance the Brillouin gain in narrow slots. We estimate a currently feasible gain of about $10^{5} \mathrm{~W}^{-1} \mathrm{~m}^{-1}$, which is an order of magnitude larger than in a stand-alone silicon wire. Such efficient coupling could enable a host of Brillouin technologies on a mass-producible silicon chip.
\end{abstract}

OCIS codes: $\quad(130.4310,190.4390)$ Nonlinear integrated optics; (290.5830) Brillouin scattering

http://dx.doi.org/10.1364/XX.99.099999

\section{Introduction}

Stimulated Brillouin scattering (SBS) is a nonlinear process which couples optical to mechanical waves $[1,2]$. It is a powerful means to control light, with applications ranging from lasing [3], comb generation [4-6] and isolation [7] to RF-waveform synthesis [8], slow/stored light $[9,10]$ and reconfigurable filtering [11]. With this in mind, SBS has been explored in a wide variety of systems, such as conventional and photonic crystal fibers [12-16], silica microspheres [17, 18] and wedge-disks [19], calcium fluoride resonators [20] and chalcogenide rib waveguides [21]. Therefore the prospect of strong SBS in small-core silicon wires [22] is tantalizing.

Such wires are known for their large Kerr and Raman nonlinearity [23]. However, Brillouin scattering has so far lagged behind in silicon. The culprit is the silica substrate on which the silicon wires are typically made. It severely decreases both the wires' mechanical flexibility and the phonons' lifetime. Unlike in chalcogenide rib waveguides [21, 24], elastic waves in silicon cannot be guided by internal reflection because sound is faster in silicon than in silica.

A theoretical model by Wang et al. [25, 26] recently predicted that the efficiency of SBS would increase dramatically by removing the substrate. Then the elastic waves are confined to the core because of the large acoustic mismatch between air and silicon, although there is still no internal reflection. The model included not just electrostriction but also radiation pressure, which was traditionally neglected as a driver of Brillouin scattering.

\footnotetext{
* raphael.vanlaer@intec.ugent.be
}

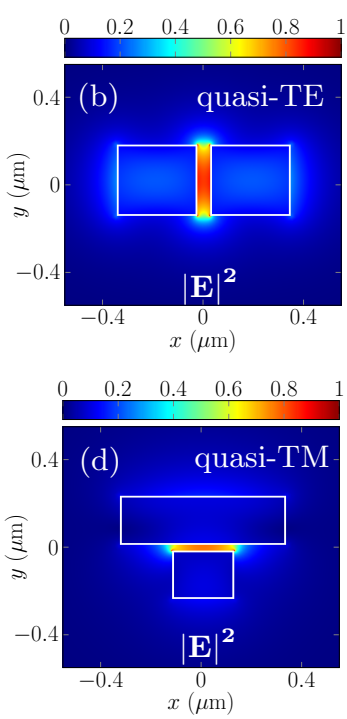

Fig. 1. Vertical (a) and horizontal (c) silicon slot waveguides suspended in air, with the corresponding optical mode $(b, d)$.

Thus electrostriction and radiation pressure interfere in nanoscale waveguides, connecting the fields of Brillouin scattering and optomechanics [27-30]. The validity of the new SBS model has been confirmed by recent observations of SBS in a hybrid silicon nitride-silicon waveguide [31], although the enhancement of SBS in silicononly photonic wires $[25,26]$ remains unverified.

In this Letter we take the study of Brillouin scattering to silicon slot waveguides, to exploit their strong mode confinement $[32,33]$ and large gradient forces [34]. We perform full-vectorial coupled optical and mechanical simulations of the Brillouin gain coefficient using the finite-element solver COMSOL. 


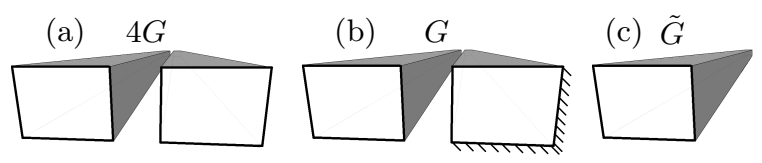

Fig. 2. We compare three scenarios: (a) a slot with two free silicon beams, (b) a slot with one free and one fixed beam and (c) a stand-alone free beam.
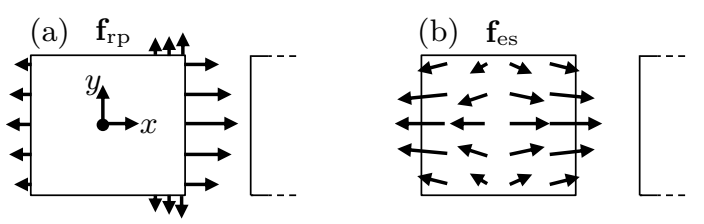

Fig. 3. Typical optical force profile on left beam of vertical slot waveguide: (a) radiation pressure and (b) electrostrictive body force. The radiation pressure is large close to the slot.

\section{Background and assumptions}

We consider vertical (fig.1a-b) and horizontal (fig.1cd) slot waveguides suspended in air. Both waveguides strongly confine light, creating large radiation pressure close to the slot. This gives rise to the possibility of (1) improving the photon-phonon coupling, (2) testing SBS theory in a regime dominated by gradient forces and (3) exciting new types of phonons.

If the two silicon beams would be identical (fig.2a), their mechanical resonances could be addressed simultaneously. Then the SBS gain would be $4 G$, with $G$ the single-beam gain. However, our simulations show that fabrication imperfections on the order of nanometers are sufficient to shift the phonon spectrum by more than one mechanical linewidth $(\sim 10 \mathrm{MHz})$. So we assume just one beam of dimensions $(a, b)$ contributes to SBS (fig.2b). Moreover, we call $\tilde{G}$ the peak gain associated with a phonon in a stand-alone silicon wire (fig.2c).

A particular mechanical mode with displacement $\mathbf{u}$, wavevector $K$, stiffness $k_{\text {eff }}$ and quality factor $Q$ has a peak SBS gain $G$ of $\omega Q|\langle\mathbf{f}, \mathbf{u}\rangle|^{2} /\left(2 k_{\text {eff }}\right)$, with $\omega$ the optical frequency, $\mathbf{f}=\mathbf{f}_{\mathrm{rp}}+\mathbf{f}_{\mathrm{es}}$ the power-normalized optical force distribution and $\langle\mathbf{f}, \mathbf{u}\rangle=\int \mathbf{f}^{*} \cdot \mathbf{u} d A$ the photonphonon overlap $[25,26]$. The radiation pressure $\mathbf{f}_{\mathrm{rp}}$ is located on the waveguide boundaries (fig.3a), while the electrostrictive force $\mathbf{f}_{\mathrm{es}}$ has both a body (fig.3b) and a boundary (not shown) component. The boundary component of $\mathbf{f}_{\mathrm{es}}$ is an order of magnitude smaller than $\mathbf{f}_{\mathrm{rp}}$. Furthermore, we define $G_{\mathrm{rp}}$ and $G_{\mathrm{es}}$ as the SBS gain when only $\mathbf{f}_{\mathrm{rp}}$ or $\mathbf{f}_{\mathrm{es}}$ is present. The total gain $G$ is determined by interference between $\mathbf{f}_{\mathrm{rp}}$ and $\mathbf{f}_{\mathrm{es}}$.

In forward (backward) SBS, the Stokes and pump wave co- (counter-) propagate. Phase-matching then requires that $K \approx 0(K \approx 2 \beta)$, with $\beta$ the pump wavevector. We launch the Stokes and pump waves into the same mode, leaving inter-modal SBS [7] for further study. In addition, we work at $\lambda=1.55 \mu \mathrm{m}$ and use a flat $Q$ of $10^{3}$ as in $[25,26]$.

\section{SBS in vertical slot waveguides}

Figures 4a-c show the forward and backward SBS spectrum for a vertical slot waveguide with dimensions
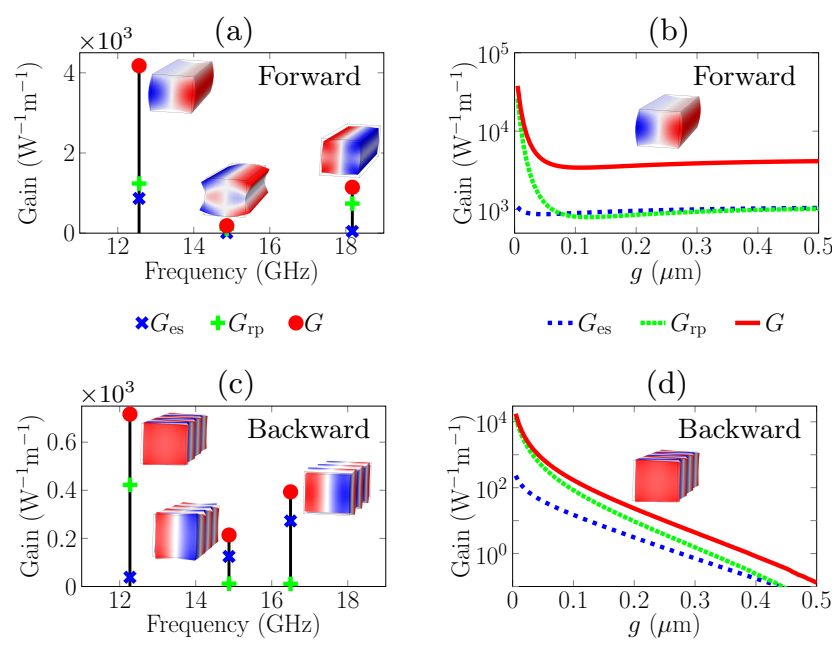

$\cdots G_{\text {es }} \quad G_{\text {rp }} \quad-G$

(d)

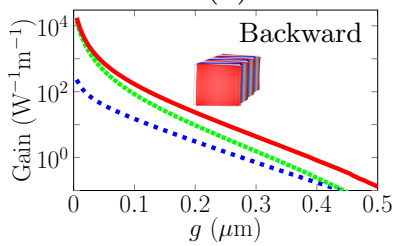

Fig. 4. (a-c) Brillouin spectrum of a vertical slot waveguide and (b-d) the gain of the most promising mode increases rapidly in narrow slots. The color of the modes indicates the sign of $u_{x}$ (red: + , blue: - ).

$(a, b, \bar{a}, g)=(315 \mathrm{~nm}, 0.9 a, a, 50 \mathrm{~nm})$, including only the three modes with largest gain.

In the forward case (fig.4a), the mechanical modes are identical to those of a stand-alone wire. The maximum gain among all modes is $4.2 \times 10^{3} \mathrm{~W}^{-1} \mathrm{~m}^{-1}$. This is smaller than $\tilde{G}=1.7 \times 10^{4} \mathrm{~W}^{-1} \mathrm{~m}^{-1}[26]$, despite the increase in radiation pressure close to the slot. The cause is a decrease in the pressure on the far side from the slot (fig.3a). If these two effects would perfectly balance, we would expect $G=\tilde{G} / 4$. This explains why $G \approx \tilde{G} / 4$ in slots as narrow as $50 \mathrm{~nm}$. Hence, smaller gaps are necessary to boost $G$ substantially. Indeed, for the most promising mode we numerically find that $G \propto 1 / g$ as $g$ falls below $50 \mathrm{~nm}$ (fig.4b). Eventually $G$ approaches a maximum of $\approx 1.1 \times 10^{5} \mathrm{~W}^{-1} \mathrm{~m}^{-1}$ as $g \rightarrow 0$. In wide slots, the optical mode evolves into the symmetric supermode of two weakly coupled silicon wires. Therefore $G \rightarrow \tilde{G} / 4$ as $g \rightarrow \infty$.

In the backward case, the mechanical modes are different from those of a stand-alone wire since the phonon wavevector $K \approx 2 \beta$ depends on the effective index $n_{p}$ of the optical mode. From the point of view of a single beam, horizontal symmetry is broken by the slot waveguide. So modes that were previously forbidden by symmetry can have non-zero gain in the slot waveguide. Such a previously forbidden phonon has the largest backward SBS gain in the slot waveguide (fig.4c). For $g=50 \mathrm{~nm}$, this phonon has a gain of $7.2 \times 10^{2} \mathrm{~W}^{-1} \mathrm{~m}^{-1}$. The optical forces are symmetric again in wide slots. Then this mode is forbidden, which means that $G \rightarrow 0$ as $g \rightarrow \infty$ (fig.4d). Going from wide to narrow slots, $G$ first increases exponentially, then its growth accelerates like $G \propto 1 / g^{1.6}$ and ultimately converges to a maximum of $\approx 4.5 \times 10^{4} \mathrm{~W}^{-1} \mathrm{~m}^{-1}$ as $g \rightarrow 0$.

In general, gradient forces dominate the SBS gain in narrow slots (fig.4b-d). The slot enhances these forces despite the reduced dispersion in such waveg- 

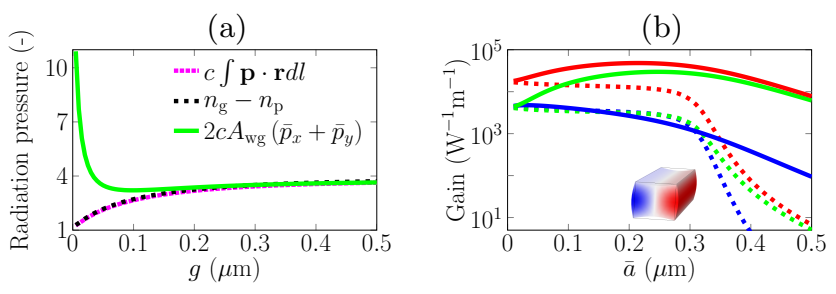

(c)
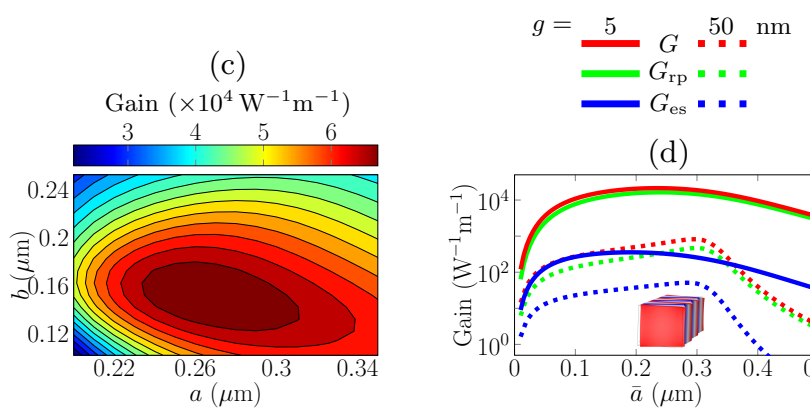

(d)

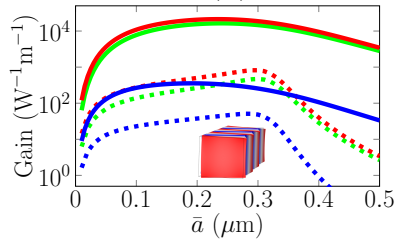

Fig. 5. (a) Gradient forces can be large despite low dispersion, (b-d) Narrow slots perform better than a stand-alone wire for a range of $\bar{a}$-values and (c) $G$ has a clear optimum in the $(a, b)$-plane for the same mode as in (b) with $g=5 \mathrm{~nm}$.

uides. As $g \rightarrow 0$, the group and effective indices $n_{g}$ and $n_{p}$ approach those of a single wire of width $a+\bar{a}$. Thus the waveguide dispersion decreases (fig.5a), contrary to the prediction that very dispersive waveguides are optimal for large gradient forces [35]. Writing the gradient force density as $\mathbf{p} \delta\left(\mathbf{r}-\mathbf{r}_{\partial \mathrm{wg}}\right)$, it was shown that $c \int \mathbf{p} \cdot \mathbf{r} d l=n_{\mathrm{g}}-n_{\mathrm{p}}$ from the scale-invariance of Maxwell's equations [35]. For a stand-alone wire the integral becomes $\int \mathbf{p} \cdot \mathbf{r} d l=A_{\mathrm{wg}}\left(\bar{p}_{x}+\bar{p}_{y}\right)$ with $A_{\mathrm{wg}}=a b$ and $\bar{p}$ the magnitude of the spatially averaged radiation pressure. However, this no longer holds for a slot waveguide. Then the integral yields $\int \mathbf{p} \cdot \mathbf{r} d l=A_{\mathrm{g}}\left(\bar{p}_{x, L}-\bar{p}_{x, R}\right)+2 A_{\mathrm{wg}}\left(\bar{p}_{x, L}+\bar{p}_{y}\right)$, with $\bar{p}_{x, L / R}$ the pressure on the left/right boundary, $A_{g}=g b$ and $a=\bar{a}$. Since $A_{\mathrm{g}} \rightarrow 0$ as $g \rightarrow 0, \bar{p}_{x, R}$ and thus $\bar{p}_{x}+\bar{p}_{y}$ can increase drastically in narrow slots (fig.5a).

Next, we investigate the effect of $\bar{a}$ (fig.5b-d). As $\bar{a} \rightarrow 0$, there is no slot-enhancement. Then $G \rightarrow \tilde{G}$, regardless of all other parameters. Furthermore, the optical mode increasingly retreats into the widest beam. This implies $G \rightarrow 0$ when $\bar{a} \rightarrow \infty$, although this effect is more pronounced in wider slots.

In the forward case (fig.5b), $\bar{a}$ affects only the force distribution. The gain $G(\bar{a})$ has a maximum in narrow slots, but decreases monotonically otherwise. This confirms that small gaps are required for substantial SBS gain enhancement in vertical slot waveguides.

In the backward case (fig. $5 \mathrm{~d}$ ), $G(\bar{a})$ always has a maximum because this phonon is forbidden in a stand-alone wire. However, the maximum increases by a factor 26 when the slot is narrowed from $50 \mathrm{~nm}$ to $5 \mathrm{~nm}$. The gain is dominated by gradient forces regardless of $(\bar{a}, g)$.

Last, we scan $(a, b)$ with $\bar{a}=a$ and $g$ fixed at $5 \mathrm{~nm}$. These parameters influence both the optical and mechanical mode. The $(a, b)$-optimum depends heavily on the slot size and on the mechanical mode. Nonethe- less, fig.5c shows that there actually exists such an optimum. We find a maximum gain of $7.0 \times 10^{4} \mathrm{~W}^{-1} \mathrm{~m}^{-1}$ for $(a, b)=(260,150) \mathrm{nm}$.

\section{SBS in horizontal slot waveguides}

The horizontal slot (fig.1c-d) has the potential advantage of (1) the extra degree of freedom $\bar{b}$ and (2) smaller gaps. In such a slot, $g$ is not limited by the resolution of lithography techniques. As a result, SBS enhancement may be within reach of current technology. As long as $\bar{b}=b$, the horizontal slot waveguide is but a rotated version of the vertical one. Therefore we immediately explore the case $\bar{b} \neq b$. We calculate the forward and backward Brillouin spectrum for a horizontal slot waveguide with dimensions $(a, b, \bar{a}, \bar{b}, g)=(160,620, a, 240,5) \mathrm{nm}$.

In the forward case (fig.6a), the fundamental flexural mode couples most efficiently. This mode has negligible SBS gain in a stand-alone wire because of cancellations in the photon-phonon overlap. Indeed, the $u_{y}$ component has two nodes, while the $y$-component of the gradient force does not change sign. Owing to $b>\bar{b}$, the cancellations can be avoided by confining the optical mode between the nodes of $u_{y}$.

In the backward case (fig.6c), there are two modes with enhanced SBS gain. The first mode has a nearly uniform $u_{y}$ component. It is a rotated version of the mode we previously studied in fig.4-5d. The second mode is the fundamental flexural mode, but at the operating point $K \approx 2 \beta$ in its dispersion diagram.

The gain increases by four orders of magnitude when $g$ drops from 250 to $5 \mathrm{~nm}$ (fig.6b). This radical enhancement is superexponential in $g$ for gaps below $50 \mathrm{~nm}$. The forward (backward) gain approaches $\approx$ $1.3 \times 10^{6} \mathrm{~W}^{-1} \mathrm{~m}^{-1}\left(1.5 \times 10^{5} \mathrm{~W}^{-1} \mathrm{~m}^{-1}\right)$ as $g \rightarrow 0$. At $g=70 \mathrm{~nm}$, an optical mode anti-crossing causes a dip in the SBS gain. However, $G(g)$ quickly recovers its original path as $g$ leaves the anti-cross region. We only show the total gain $G$ because $G_{\text {es }}$ is at least a factor $10^{5}$ $\left(10^{2}\right)$ smaller than $G_{\mathrm{rp}}$ across the entire sweep range in the forward (backward) case. Thus SBS by these modes is driven by gradient forces only, with a vanishing electrostrictive contribution.

Finally, we sweep $\bar{b}$ (fig.6d). In the forward case, $k_{\text {eff }}$ and $\mathbf{u}$ do not depend on $\bar{b}$. Then we explore purely the effect of the gradient force density $\mathbf{f}_{\mathrm{rp}}(\bar{b})$ on the photonphonon overlap $\left\langle\mathbf{f}_{\mathrm{rp}}(\bar{b}), \mathbf{u}\right\rangle$. The coupling is optimal for $\bar{b}=240 \mathrm{~nm}$. For smaller $\bar{b}, G$ decreases because the slotenhancement occurs only in a small region. For larger $\bar{b}, G$ decreases because the optical mode is no longer confined between the nodes of $u_{y}$. In the backward case, the operating point $K \approx 2 \beta$ changes as $n_{p}$ depends on $\bar{b}$. This propagating phonon is less sensitive to $\bar{b}$ because of its nearly uniform $u_{y}$ component.

\section{Conclusion}

To conclude, we found that strong gradient forces improve the efficiency of Brillouin scattering in narrow silicon slot waveguides. However, appreciable enhancement 

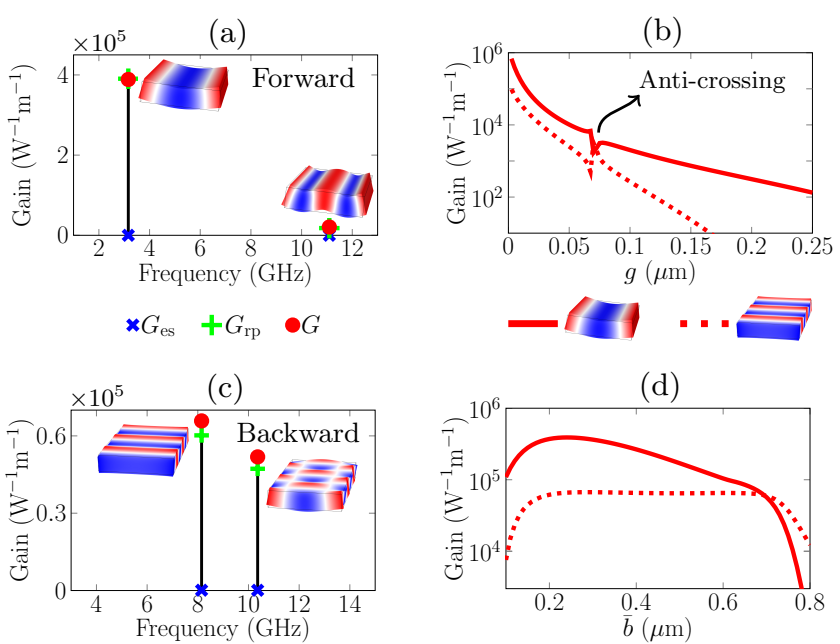

Fig. 6. (a-b-c) Both forward and backward SBS is very efficient in narrow horizontal slots and $(d)$ the flexural mode is sensitive to $\bar{b}$. The color of the modes indicates the sign of $u_{y}$ (red: + , blue: - ).

compared to a stand-alone wire is currently only accessible in horizontal slots. In such slots, we expect very efficient SBS because (1) small gaps should be technologically feasible and (2) the fundamental mechanical flexural mode can be excited. The suspension of long silicon beams remains the most important hurdle towards testing these predictions. A practical device may consist of a disconnected series of such waveguides as in [31].

\section{Supplementary information}

We use isotropic elasticity coefficients $\left(c_{11}, c_{12}, c_{44}\right)=$ $(217,85,66) \mathrm{GPa}$ for easy comparison with [25, 26]. Silicon is mechanically anisotropic, so in a more accurate calculation the coefficients $\left(c_{11}, c_{12}, c_{44}\right)=$ $(166,64,80) \mathrm{GPa}$ should be used for a guide along a $\langle 100\rangle$ crystal axis [36]. Further, we use the photoelastic coefficients $\left(p_{11}, p_{12}, p_{44}\right)=(-0.094,0.017,-0.051)[37]$, which is also valid in case the guide is aligned along a $\langle 100\rangle$ axis. We perform our calculations using the weakform [38] COMSOL module with the MATLAB Livelink.

\section{Acknowledgement}

R.V.L. acknowledges the Agency for Innovation by Science and Technology in Flanders (IWT) for a $\mathrm{PhD}$ grant. This work was partially funded under the FP7ERC-InSpectra programme. R.V.L. thanks Thomas Van Vaerenbergh for helpful discussions.

\section{References}

[1] R. Chiao, C. Townes, and B. Stoicheff, Phys. Rev. Lett. (1964).

[2] Y. Shen and N. Bloembergen, Phys. Rev. 290 (1965).

[3] L. Stokes, M. Chodorow, and H. Shaw, Opt. Lett. 7, 509 (1982).

[4] D. Braje, L. Hollberg, and S. Diddams, Phys. Rev. Lett. 102, 1 (2009).

[5] M. Kang, A. Nazarkin, A. Brenn, and P. Russell, Nature Phys. 5, 276 (2009).

[6] A. Savchenkov, A. Matsko, V. Ilchenko, D. Seidel, and L. Maleki, Opt. Lett. 36, 3338 (2011).
[7] M. Kang, A. Butsch, and P. Russell, Nature Photon. 5, 549 (2011).

[8] J. Li, H. Lee, and K. Vahala, Nat. Commun. 4, 1 (2013).

[9] Y. Okawachi, M. Bigelow, J. Sharping, Z. Zhu, A. Schweinsberg, D. Gauthier, R. Boyd, and A. Gaeta, Phys. Rev. Lett. 153902, 1 (2005).

[10] Z. Zhu, D. Gauthier, and R. Boyd, Science 318, 1748 (2007).

[11] A. Zadok, A. Eyal, and M. Tur, J. Lightw. Technol. 25, 2168 (2007).

[12] E. Ippen and R. Stolen, Appl. Phys. Lett. 21, 539 (1972).

[13] R. Shelby, M. Levenson, and P. Bayer, Phys. Rev. Lett. 54, 939 (1985).

[14] A. Kobyakov, M. Sauer, and D. Chowdhury, Adv. Opt. and Photon. 2, 1 (2009).

[15] J. Wang, Y. Zhu, R. Zhang, and D. Gauthier, Opt. Express 19, 5339 (2011).

[16] J. Beugnot, T. Sylvestre, and H. Maillotte, Opt. Lett. 32, 2006 (2007).

[17] M. Tomes and T. Carmon, Phys. Rev. Lett. 102, 113601 (2009).

[18] G. Bahl, J. Zehnpfennig, M. Tomes, and T. Carmon, Nat. Commun. 2, 403 (2011).

[19] J. Li, H. Lee, T. Chen, and K. Vahala, Opt. Express 20, 369 (2012).

[20] I. Grudinin, A. Matsko, and L. Maleki, Phys. Rev. Lett. 102, 043902 (2009).

[21] R. Pant, C. Poulton, D. Choi, H. Mcfarlane, S. Hile, E. Li, L. Thevenaz, B. Luther-Davies, S. Madden, and B. Eggleton, Opt. Express 19, 8285 (2011).

[22] W. Bogaerts, R. Baets, P. Dumon, V. Wiaux, S. Beckx, D. Taillaert, B. Luyssaert, J. Van Campenhout, P. Bienstman, and D. Van Thourhout, J. Lightw. Technol. 23, 401 (2005).

[23] Q. Lin, O. Painter, and G. Agrawal, Opt. Express 15, 416 (2007).

[24] C. Poulton, R. Pant, and B. Eggleton, J. Opt. Soc. Am. B , 1 (2013).

[25] P. Rakich, C. Reinke, R. Camacho, P. Davids, and Z. Wang, Phys. Rev. X 2, 1 (2012).

[26] W. Qiu, P. Rakich, M. Soljacic, and Z. Wang, arXiv preprint arXiv:1210.0267 5865, 2764 (2012).

[27] T. Kippenberg and K. Vahala, Opt. Express (2007).

[28] T. Kippenberg and K. Vahala, Science 321, 1172 (2008).

[29] M. Li, W. Pernice, C. Xiong, T. Baehr-Jones, M. Hochberg, and H. Tang, Nature 456, 480 (2008).

[30] D. Van Thourhout and J. Roels, Nature Photon. 4, 211 (2010).

[31] H. Shin, W. Qiu, R. Jarecki, J. Cox, R. Olsson, A. Starbuck, Z. Wang, and P. Rakich, Nat. Commun. 4, 1944 (2013).

[32] V. Almeida, Q. Xu, C. Barrios, and M. Lipson, Opt. Lett. 29, 1209 (2004).

[33] R. Sun, P. Dong, N. Feng, C. Hong, J. Michel, M. Lipson, and L. Kimerling, Opt. Express 15, 17967 (2007).

[34] M. Li, W. Pernice, and H. Tang, Appl. Phys. Lett. 97, 183110 (2010).

[35] P. Rakich, Z. Wang, and P. Davids, Opt. Lett. 36, 217 (2011).

[36] M. Hopcroft, W. Nix, and T. Kenny, J. Microelectromech. Syst. 19, 229 (2010).

[37] D. Biegelsen, Phys. Rev. Lett. D (1974).

[38] S. Dasgupta and F. Poletti, J. Lightw. Technol. 29, 22 (2011). 